\title{
Numerical techniques for simulating groundwater flow in the presence of temperature gradients
}

\author{
Irene Pestov* \\ (Received 7 August 2000)
}

\begin{abstract}
In this paper we are concerned with situations when vertical temperature gradients in a groundwater aquifer exceed a value determined by the critical Rayleigh number for the onset of thermal convection. In the presence of such temperature gradients, the groundwater flow
\end{abstract}

*Bureau of Rural Sciences, Canberra, ACT 2601, Australia. mailto:irene.pestov@brs.gov.au

${ }^{0}$ See http: //anziamj . austms .org. au/V42/CTAC99/Pest for this article and ancillary services, (c) Austral. Mathematical Soc. 2000. Published 27 Nov 2000. 
exists in the form of convective circulation. The variation of water density triggers thermal convection and the variation of dynamic viscosity enhances flow within convective cells. We discuss the mathematical and numerical framework of convective groundwater flow and examine the effects of anisotropic rock permeability and large-scale inhomogeneities on the convective motion.

\section{Contents}

1 Introduction

C1116

2 The Rayleigh Number

C1117

3 A Mathematical Model

C1120

4 Numerical Examples

C1122

5 Conclusions

C1131

References

C1132 


\section{Introduction}

In an aquifer heated from below warmer and lighter water layers are overlaid by cooler and denser ones. When the vertical temperature difference is small, this configuration remains stable due to water viscosity. At some temperature difference, the buoyancy forces overcome stabilising effects of the viscous forces and instability appears in the form of a convective motion. The lighter fluid flows up driven by buoyancy, and the denser fluid moves down driven by gravity. The groundwater flow forms large-scale rolls or convective cells. Both density and viscosity variations with temperature are important for the formation of the convective cells: density variation triggers the onset of convection and viscosity variation enhances the flow within the cells.

Because of its practical importance, natural convection in porous media has attracted considerable interest in scientific literature. Now it is an established area of applied mathematics with a well developed theoretical foundation and state-of-the-art computer software available [3, 14, 18, 5].

The early works in this area were devoted to determining quantitative criteria for the onset of thermal convection from linearised governing equations. The critical Rayleigh number was first calculated in [7] for a homogeneous porous medium saturated by a single-phase fluid. This work was further advanced in $[10,11,12,13]$ through incorporating more realistic features such as inhomogeneity and anisotropy of a porous medium. Linear stability problems of two-phase liquid-vapour thermal convection in porous media were consid- 
ered by numerous authors, mainly in works related to geothermal reservoir modelling, cf. [1, 19, 20, 9, 15]. Convection induced by concentration gradients of dissolved salts was first investigated in [21]. Although the latter is of considerable interest to groundwater aquifer modelling, we leave this type of natural convection beyond the scope of this paper. A comprehensive review of works on natural convection in porous media is given in $[3,14]$.

\section{The Rayleigh Number}

The Rayleigh number is the main non-dimensional number for thermal convection problems. It includes characteristics of a porous medium, thermodynamic properties of a saturated fluid and a vertical temperature difference. In simplified cases, the critical Rayleigh number for the onset of convection can be calculated from linearised governing equations. In more complex cases laboratory or computer experiments are required.

In a homogeneous isotropic porous medium saturated by a single-phase fluid the Rayleigh number is defined as

$$
\mathrm{Ra}=\frac{\rho g \beta k H \Delta T}{\mu \alpha},
$$

where $\rho$ and $\mu$ are fluid density and dynamic viscosity respectively, $g$ is gravitational acceleration, $\beta$ is thermal expansion coefficient of the fluid, $k$ is permeability, $\alpha$ is thermal diffusivity of the saturated porous medium, 
and $\Delta T$ is the temperature difference over a distance $H$. In equation (1) $H$ and $\Delta T$ are positive in the direction of $g$. A critical Rayleigh number for a homogeneous isotropic porous medium bounded from above and below is [7]:

$$
\mathrm{Ra}^{c r}=4 \pi^{2} \approx 39.48 .
$$

For $\mathrm{Ra}<\mathrm{Ra}^{c r}$ the heat transfer in the porous medium occurs solely by thermal conduction and the conduction state remains stable. When

$$
\mathrm{Ra}>\mathrm{Ra}^{c r}
$$

instability appears in the form of circulatory flows.

Often a groundwater aquifer consists of several layers of different permeability and should not be considered as homogeneous and isotropic. Nondimensional criteria for the onset of thermal convection in layered systems have been developed in $[10,11,13]$. In these works the Rayleigh number is calculated using permeability and thermal properties of the bottom layer and the temperature difference and thickness of the whole system. In the case of a stratified system which consists of a large number of thin layers, the Rayleigh number is based on the effective vertical permeability and conductivity calculated using an averaging procedure [12]. The multilayered structure markedly affects the critical Rayleigh number. For a three-layer system the values of the critical Rayleigh number are increasing with decreasing permeability of the middle layer [10]. In [10] the middle layer occupied $20 \%$ of the total aquifer thickness and had a permeability $k_{1}$ different to that of the top and bottom layers. (The top and bottom layers had equal permeability $k_{2}$.) The calculated values of $\mathrm{Ra}^{c r}$ were $92.14,188.08$ and 194.23 for 
$k_{1} / k_{2}=0.1,0.01$ and 0.001 respectively. Even for moderate geothermal gradients found in Australia, Rayleigh numbers may exceed these critical values, so that inequality (3) will be satisfied [16].

It is not possible to obtain critical Rayleigh numbers for all structural configurations of soils found in real systems. However, as a preliminary step it is useful to calculate the Rayleigh number given by equation (1). For largescale heterogeneous systems the Rayleigh number at each grid point can be conveniently evaluated using the GIS tools. For instance, with the help of ArcView Spatial Analyst, corresponding grids of the aquifer thickness $(H)$, geothermal gradient $(\Delta T)$ and vertical permeability $(k)$ can be multiplied in accordance with equation (1), and a new grid coverage for Ra can be obtained. After that, inequality (3) can be applied to identify regions where Ra exceeds the critical value for a homogeneous isotropic porous medium given by equation (2). For complex real field situations further numerical simulations of the regional flows will be needed.

When choosing a numerical model, it is important to check that the corresponding mathematical model includes coupling between heat and mass transfer processes and that there is a dependence of water thermodynamic properties on temperature. Isothermal flow models are no longer applicable to convective flows. Since convective flows have a significant vertical component, numerical simulations must be $3 \mathrm{D}$ or $2 \mathrm{D}$ with the vertical coordinate included. It is not possible to describe the convective motion by horizontal flow models (e.g. via introducing leakage terms). 


\section{A Mathematical Model}

The following system of conservation equations describes non-isothermal flow in a groundwater aquifer [14].

\section{Continuity equation:}

$$
\frac{\partial}{\partial t}(\phi \rho)+\nabla \cdot(\rho \mathbf{V})=W
$$

\section{Energy equation:}

$$
\left[(1-\phi) \rho_{s} C_{s}+\phi \rho C_{p}\right] \frac{\partial T}{\partial t}+\rho_{l} C_{p} \mathbf{V} \cdot \nabla T=\nabla \cdot(\lambda \nabla T),
$$

Darcy's law:

$$
\mathbf{V}=-\frac{k}{\mu}\left(\nabla p-\rho g \mathbf{e}_{z}\right)
$$

Here $\phi$ is porosity, $\rho_{s}$ is solid phase density, $C_{p}$ is specific heat of water at constant pressure, $\mathbf{V}$ is Darcy velocity vector, $\mathbf{e}_{z}$ is the unit vector in the direction of the gravity force, $C_{s}$ and $\lambda$ are specific heat and thermal conductivity of the saturated porous medium respectively, and $W$ is the volumetric source term. For the mathematical model to be complete, it is necessary to add to equations (4-6) constitutive relationships describing thermodynamic 
properties of solid and liquid phases as functions of temperature and pressure, hydrodynamic and thermal boundary conditions, and initial conditions for time-dependent problems.

Note that the concept of potentiometric head is no longer applicable to non-isothermal flows, and equations (4-6) involve pressure $p$ and temperature $T$ as dependent variables. Equation (5) is known as the generalised Darcy's law equation. It is used to describe non-isothermal and variable density flows in porous media when the Reynolds number is less than 10 [2, 14]. The numerical program MoDFLOW [8] solves the Darcy's law equation in the following form:

$$
\mathbf{V}=-K \nabla h
$$

where $K=k \rho g / \mu$ is hydraulic conductivity and $h=p / \rho g$ is potentiometric head. Only in a very particular case where $\rho=$ const, equation (7) is identical to equation (5). Therefore, MODFLOW cannot be used to simulate convective groundwater flow. The variable density flow modification of MODFLOW [6] is not applicable either, since it ignores coupling between heat and mass transfer processes.

There is an extra level of complexity associated with incorporating thermal convection in groundwater models. Indeed, one has to solve two nonlinear partial differential equations in every grid point which could be computationally expensive, especially on large grids. However, thermal convection cannot be neglected when determining flow directions and the aquifer response to groundwater extraction as we illustrate in our numerical examples. 


\section{Numerical Examples}

We considered a rectangular aquifer cross-section $625 \mathrm{~m}$ by $575 \mathrm{~m}$ bounded from above and below by impermeable surfaces at $25 \geq z \geq 0$ and $-575 \geq$ $z \geq-600$ (Figure 1 ). There were 625 grid cells, $25 m$ by $25 m$ each. Constant temperatures $T=36^{\circ} \mathrm{C}$ and $T=60^{\circ} \mathrm{C}$ were prescribed at the upper and lower boundaries respectively. This corresponds to a geothermal gradient of $41.7 \mathrm{~K} / 1000 \mathrm{~m}$ which is slightly above the world average value of $30 \mathrm{~K} / 1000 \mathrm{~m}$ as given in [4]. Rock thermal conductivity was $3.2 \mathrm{~W} / \mathrm{m} \mathrm{K}$, rock density was $2650 \mathrm{~kg} / \mathrm{m}^{3}$, heat capacity was $1000 \mathrm{~J} / \mathrm{kg} \mathrm{K}$ and porosity was 0.20 .

In our numerical simulations the numerical package TOUGH2 was used [17]. TOUGH2 is a finite-difference numerical program that can handle coupled $2 \mathrm{D}$ and $3 \mathrm{D}$ groundwater and heat flows in porous and fractured media. It solves the continuity and energy equations $(4-5)$ together with the generalised Darcy's law (6) and includes numerical subroutines for calculating groundwater density and dynamic viscosity as functions of temperature. The latter is particularly important when non-isothermal groundwater flow is considered. (Note that MODFLOW [8] does not solve the energy equation and assumes $\rho$ and $\mu$ to be constant.)

In the first series of computer experiments we examined the effects of anisotropic permeability. We assumed the aquifer horizontal permeability $k_{x}$ to be larger than its vertical permeability $k_{z}$. Figure 1 shows calculated groundwater velocity vectors for $k_{x}=1 d$ and $k_{z}=0.5 d$. (Here 


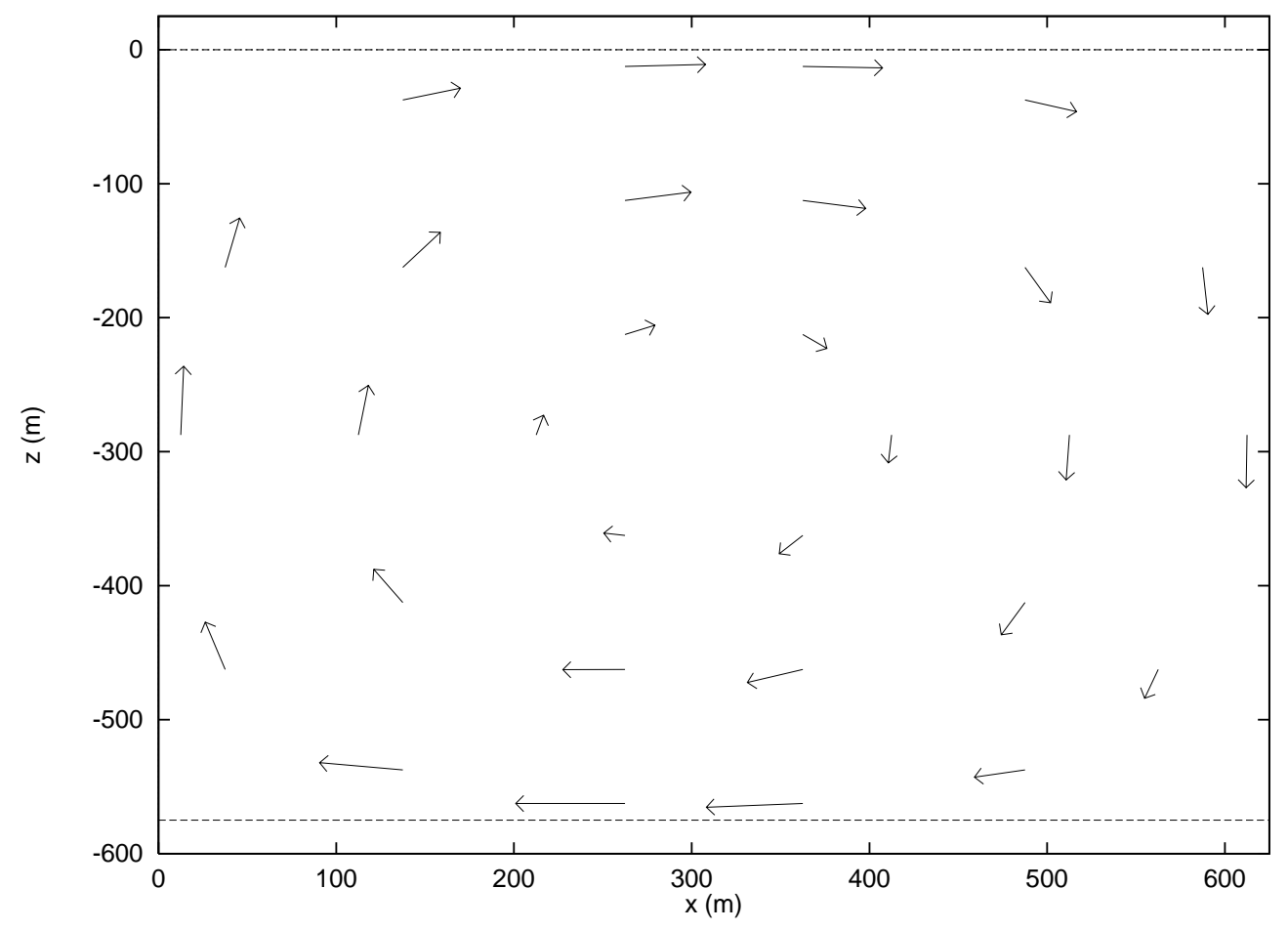

FigURE 1: Groundwater velocity vectors for $k_{x}=1 d$ and $k_{z}=0.5 d$. 


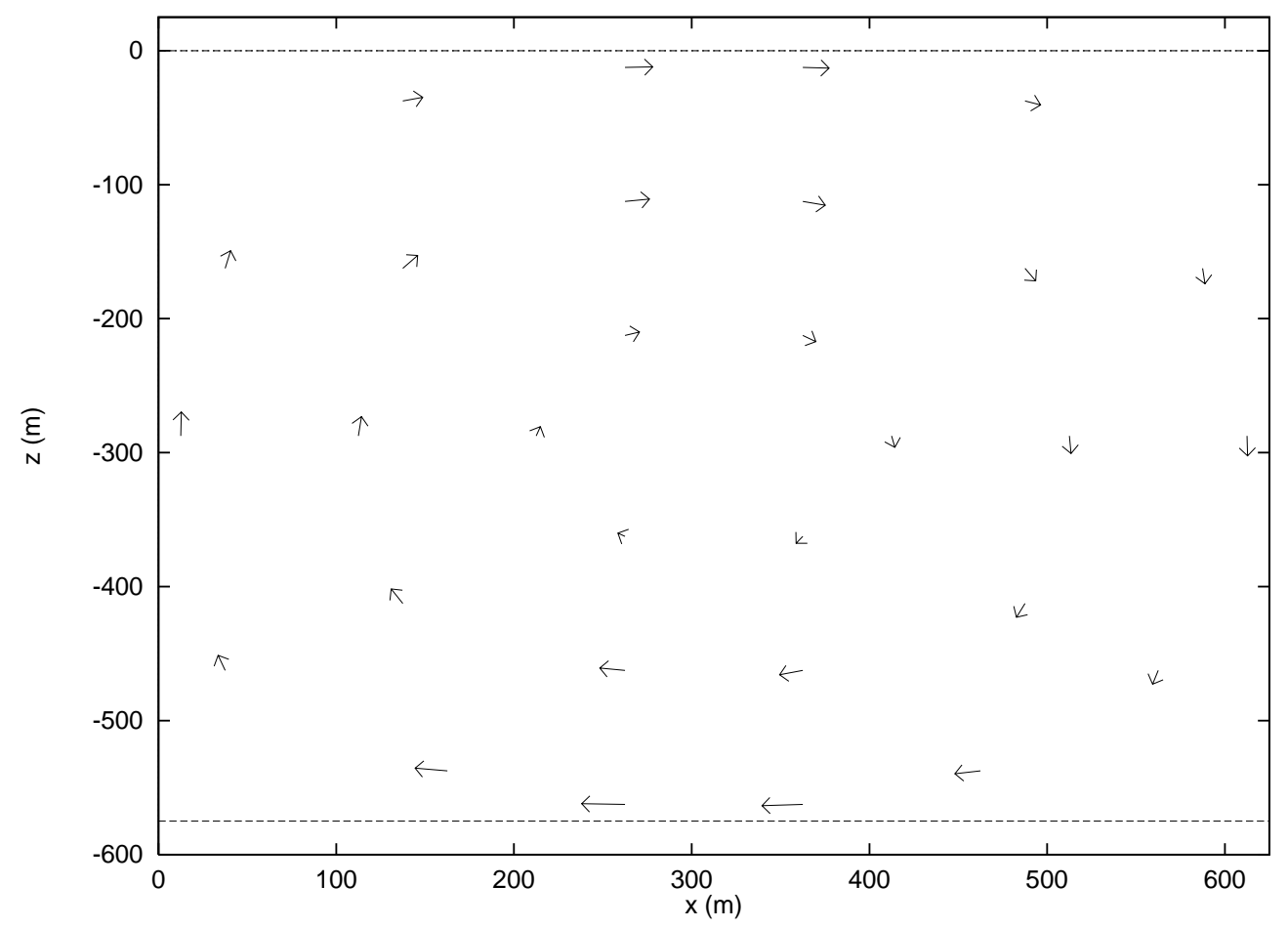

Figure 2: Groundwater velocity vectors for $k_{x}=1 d$ and $k_{z}=0.2 d$. 
$1 d=1$ darcy $=10^{-12} m^{2}$.) The magnitudes of the velocity vectors were enlarged to make the relative acceleration of groundwater flow visible. The observed groundwater velocities were of the order of $3 \times 10^{-8} \mathrm{~m} / \mathrm{s}$. A similar flow pattern, but with smaller groundwater velocities of $1.2 \times 10^{-8} \mathrm{~m} / \mathrm{s}$, was obtained for $k_{z}=0.2 d$ (Figure 2). There was no convection when $k_{z}$ was as low as $0.1 d$.

In the next series of computer experiments we examined the effects of large-scale inhomogeneities on thermal convection. We placed a $125 \mathrm{~m}$ thick aquitard in the middle of the aquifer. There were two permeable windows at the left and right side of the aquitard. The aquifer and window permeability was $500 \mathrm{md}$ and the aquitard permeability was $0.001 \mathrm{md}$. In studying thermal convection in this system, only the vertical aquifer dimension is of interest. A choice of the horizontal extent of the model could be of any reasonable value with no or little effect on a flow pattern.

The calculated groundwater velocity vectors are shown in Figure 3. Again, the magnitudes of the velocity vectors were enlarged to make them visible. Groundwater ascended through the left window driven by buoyancy and descended through the right window driven by gravity. The flow took a shape of a clockwise rotating convective cell: in the top aquifer layer water percolated from left to right and in the bottom aquifer layer the water movement was in the opposite direction. Water moved faster through the windows and there was no groundwater flow through the aquitard. The observed groundwater velocities were of the order of $1.8 \times 10^{-8} \mathrm{~m} / \mathrm{s}$. 


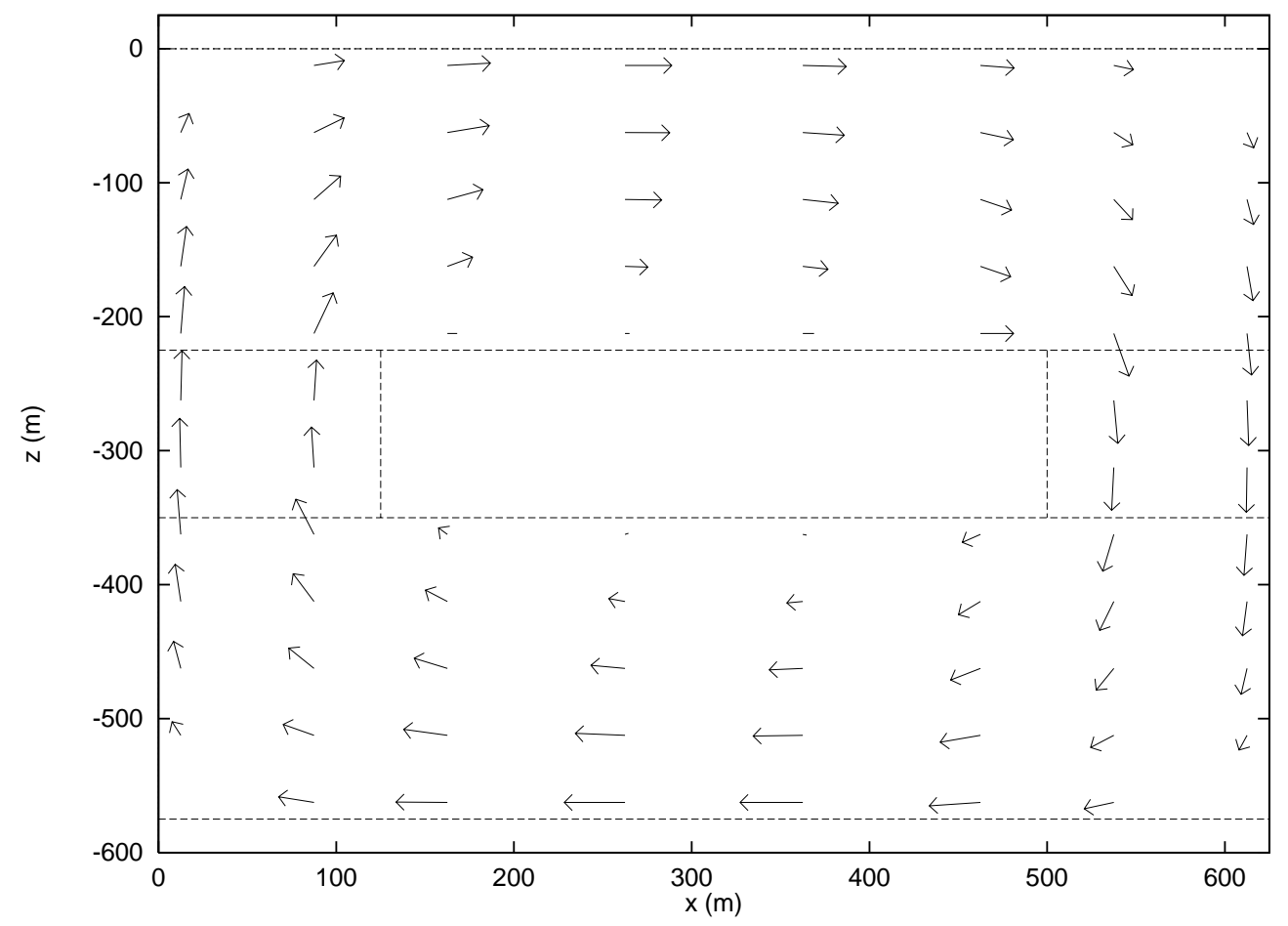

Figure 3: Groundwater velocity vectors. 
Groundwater velocities of $3 \times 10^{-8} \mathrm{~m} / \mathrm{s}$ and flow patterns similar to that of Figure 3 were obtained in an aquifer separated by an aquitard with windows when horizontal rock permeability was increased to $1 d$.

Vertical temperature and pressure distributions for various $x$ are shown in Figure 4 and Figure 5 respectively. In Figure 4 the $x$-coordinate of the vertical temperature profiles is increasing from right to left with $x=12.5$, $162,262,362,462$ and 612.5 metres, so that the rightmost and leftmost vertical temperature distributions correspond to $x=12.5 \mathrm{~m}$ (left window) and $x=612.5 \mathrm{~m}$ (right window) respectively. There were significant temperature variations in both horizontal and vertical directions. In contrast, vertical pressure profiles for all six values of $x$ coincided with the hydrostatic pressure distribution (Figure 5). The latter indicated that the groundwater flow was totally driven by heat.

Our numerical simulations have shown that only relatively large aquitard thicknesses and very low window permeabilities acting together could stop thermal convection. There was no convection in the aquifer separated by a $125 \mathrm{~m}$ thick aquitard with $k_{w}=0.1 d$ (ten times smaller than the aquifer permeability). However, we still observed thermal convection at $k_{w}=0.2 d$. We also observed the convective motion in the top and bottom aquifer layers separated by a $25 \mathrm{~m}$ thick aquitard with no windows. The observed groundwater flow formed two clock-wise rotating convective cells above and below the aquitard. The groundwater velocities were of the order of $10^{-14} \mathrm{~m} / \mathrm{s}$.

In the above examples it is not possible to obtain physically realistic 
groundwater flow lines using isothermal models.

In the last example we considered a radial flow to a water bore in a $100 \mathrm{~m}$ thick aquifer. Mass flow rates were taken to be $0,10,20,30,40$ and $50 \mathrm{~kg} / \mathrm{s}$. Water temperature in the aquifer was set to be $70^{\circ} \mathrm{C}$. There was an adjusted cold water aquifer with water temperature $T=20^{\circ} \mathrm{C}$. Under natural conditions there was no flow between two aquifers. When production started, cold water entered the hot water aquifer and water temperature of the latter decreased. This temperature decrease affected water dynamic viscosity and density and, hence, hydraulic conductivity $K$. Figure 6 shows hydraulic conductivity $K$ versus time in a grid block at a distance of $500 \mathrm{~m}$ from the water bore. Higher mass flow rates correspond to faster decays of hydraulic conductivity.

Some isothermal models include the dependence of water thermodynamic properties on temperature by calibrating hydraulic conductivity against measurements. The values of hydraulic conductivity obtained in such a manner inevitably reflect the temperature distribution existed at the time of measurements. It is obvious that the obtained values may become outdated as the real field conditions change with time. The above example illustrates aging of the isothermal model parameters. The aging is faster in regions with large numbers of water bores where the extraction rates are high. 


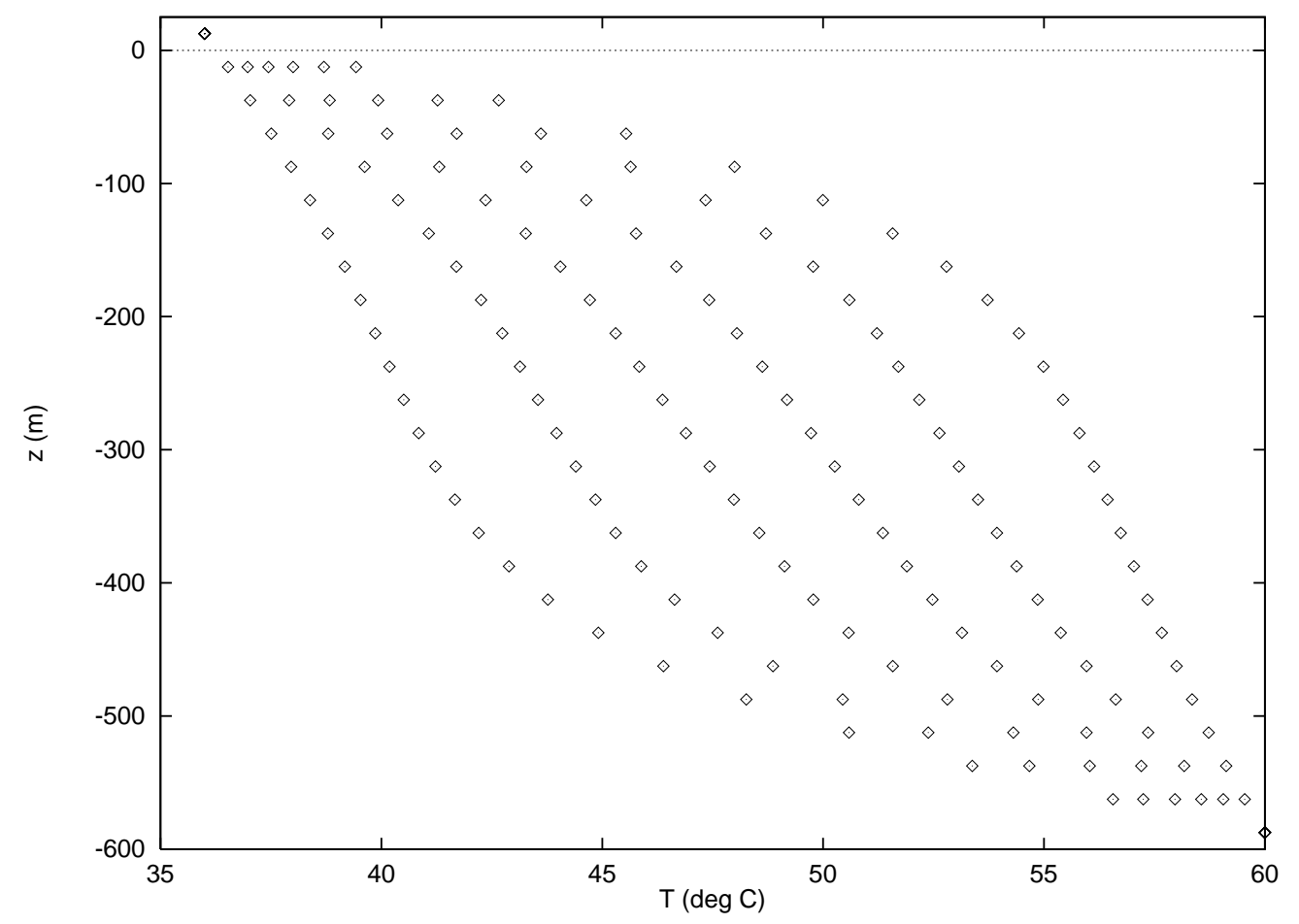

Figure 4: Temperature versus depths for various $x$. 


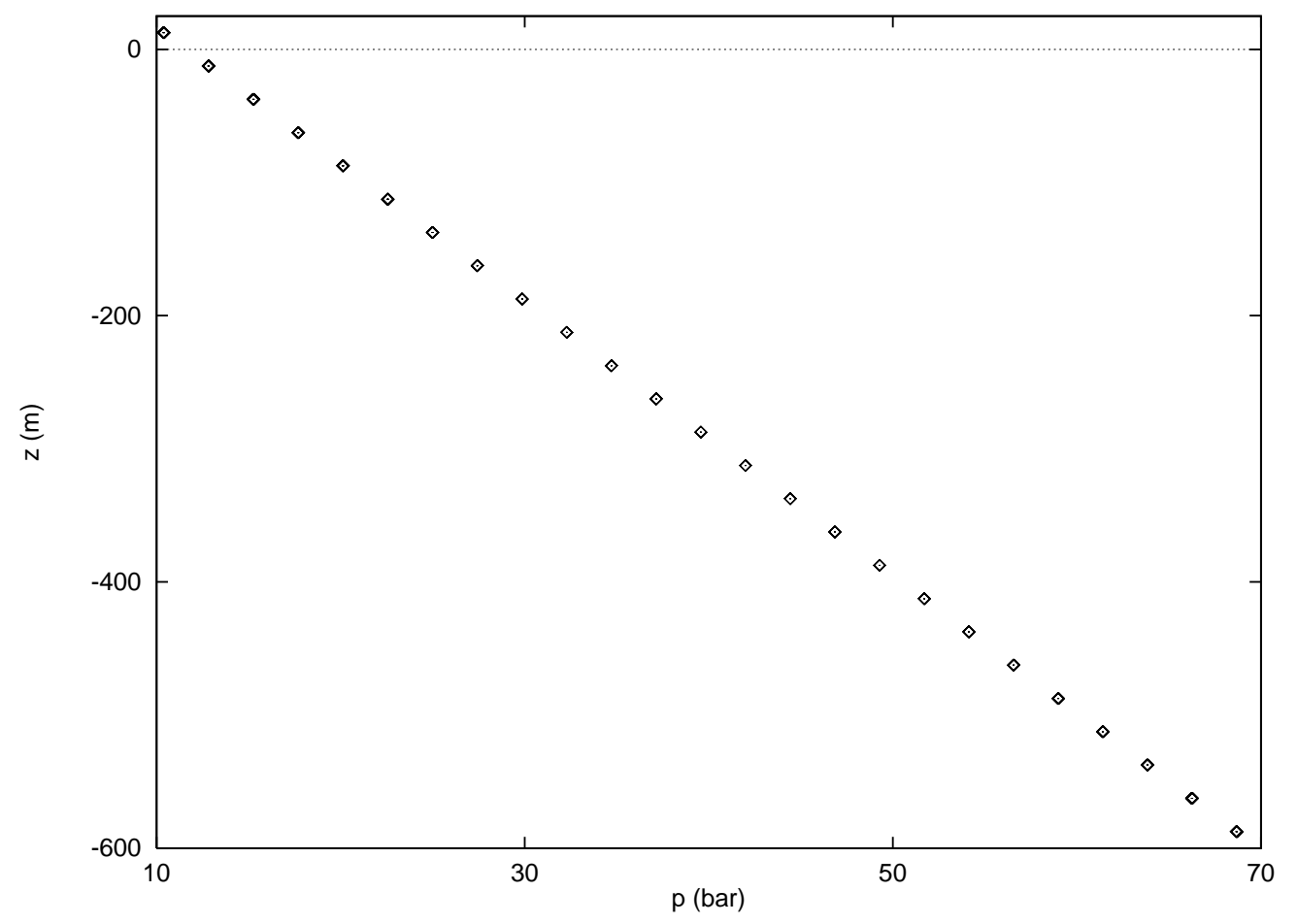

Figure 5: Pressure versus depths for various $x$. 


\section{Conclusions}

Thermal convection occurs naturally in a groundwater aquifer. Even for moderate geothermal gradients found in Australia, vertical temperature differences across aquifers may exceed a value determined by the critical Rayleigh number for the onset of thermal convection. In the presence of such temperature gradients, the groundwater flow exists in the form of large-scale convective circulation. Temperature gradients in the vertical direction, not horizontal pressure differences, become a major driving force for the groundwater flow.

It is not possible to describe the convective motion by isothermal flow models. The mathematical model of convective groundwater flow must include coupling between heat and mass transfer processes and the dependence of groundwater thermodynamic properties on temperature. As the momentum equation, the generalised Darcy's law must be used. Since the convective motion have a significant flow component in the vertical direction, 3D or 2D models with the vertical coordinate included must be applied.

Anisotropic rock permeability and large-scale inhomogeneities found in real systems affect thermal convection. Our numerical experiments have shown that large horizontal permeability increases the flow, whereas low vertical permeability and impermeable barriers reduce flow velocities. However, only very low vertical permeability of less than $0.2 d$ and relatively thick impermeable barriers of more than $5 \%$ of the total aquifer thickness stop the 
convective motion altogether. In the presence of large-scale inhomogeneities, the convective motion can form peculiar flow patterns. For instance, groundwater can flow in the opposite directions in the same aquifer layer above and below a dividing aquitard. It is not possible to simulate such a flow pattern using isothermal models.

Incorporating thermal convection in groundwater models is important for determining the flow directions and the aquifer response to groundwater extraction. The groundwater extraction scenarios considered in this paper have shown that at high extraction rates hydraulic conductivity may change by almost a factor of 2 over a period of time of just 10 years. Transient changes in hydraulic conductivity will inevitably affect the groundwater flow directions and, therefore, must be accommodated into groundwater models.

Acknowledgements: I thank Jacob Bear, Mark McGuinness, Robert McKibbin and Stephen White for helpful communication.

\section{References}

[1] H.H. Bau and K.E. Torrance. Boiling low-permeability porous material. In. J. Heat Mass Transfer, 25:45-55, 1982. C1117 


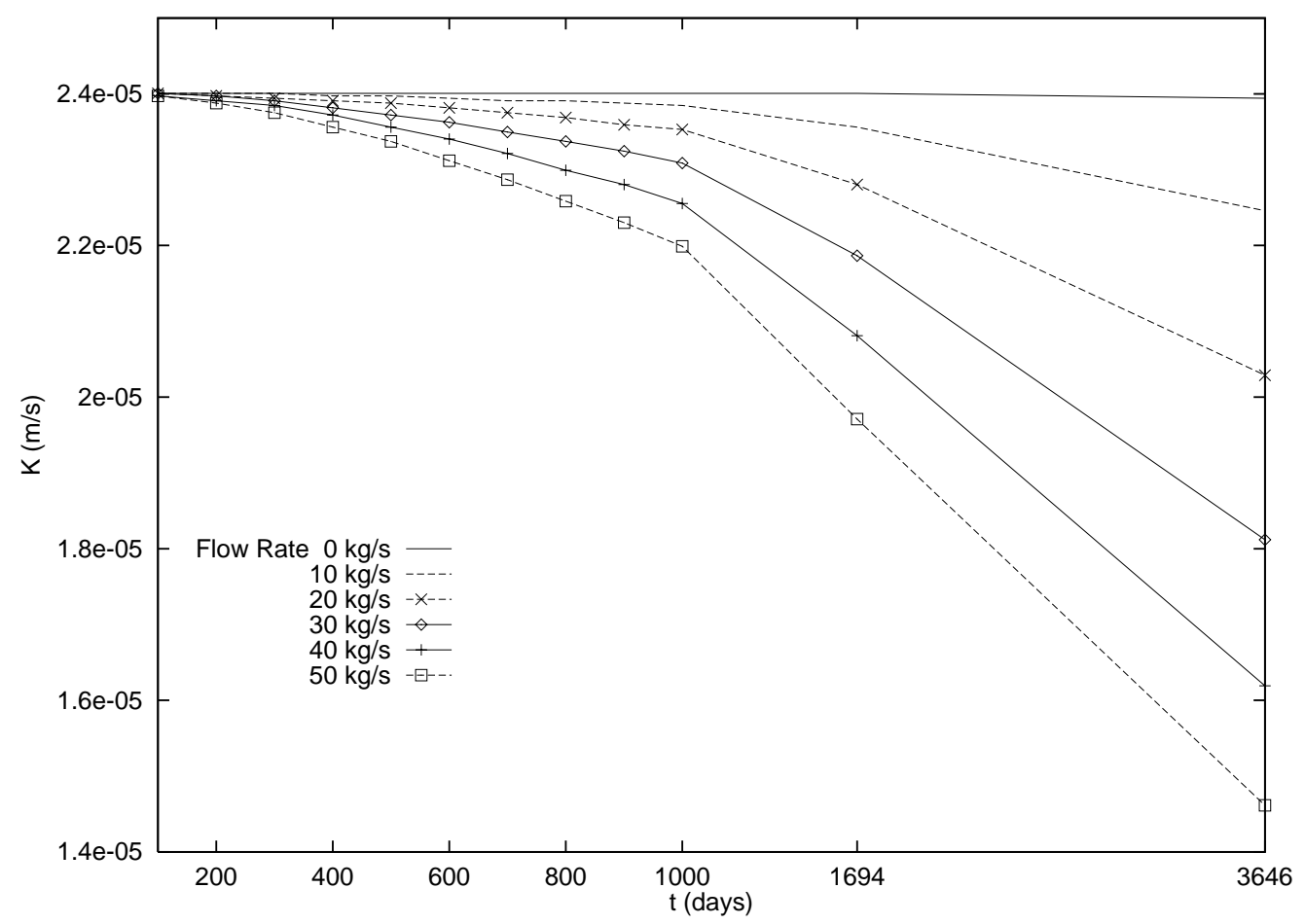

Figure 6: Hydraulic conductivity versus time. 
[2] J. Bear and A. Verruijt. Modelling Groundwater Flow and Pollution. Kluwer Academic Publishers, 1987. C1121

[3] P. Cheng. Heat transfer in geothermal systems. Adv. Heat Transfer, 14:1-105, 1978. C1116, C1117

[4] M.A. Grant, I.G. Donaldson, and P.F. Bixley. Geothermal Reservoir Engineering. Academic Press, 1982. C1122

[5] S. Finsterle and K. Pruess. Solving the estimation-identification problem in two-phase flow modeling. Water Resour. Res., 31:913-924, 1995. C1116

[6] L.K. Kuiper A numerical procedure for the solution of the steady state variable density groundwater flow equation. Water Resour. Res., 19:234-240, 1983. C1121

[7] E.R. Lapwood. Convection of a fluid in a porous medium. Proc. Cambridge Phil. Soc., 44:508-521, 1948. C1116, C1118

[8] M.G. McDonald and A. Harbaugh. A modular Three-Dimensional Finite-Difference Ground-Water Flow Model. US Government Printing Office, 1988. C1121, C1122

[9] M.J. McGuinness. Heat pipe stability in geothermal reservoirs. Geother. Resour. Council Trans., 14:1301-1307, 1990. C1117 
[10] R. McKibbin and M.J. O'Sullivan. Onset of convection in a layered porous medium heated from below. J. Fluid Mech., 96:375-393, 1980. C1116, C1118, C1118, C1118

[11] R. McKibbin and M.J. O'Sullivan. Heat transfer in a layered porous medium heated from below. J. Fluid Mech., 111:141-173, 1981. C1116, C1118

[12] R. McKibbin and P.A. Tyvand. Anisotropic modelling of thermal convection in multilayered porous media. J. Fluid Mech., 118:315-339, 1982. C1116, C1118

[13] R. McKibbin and P.A. Tyvand. Thermal convection in a porous medium composed of alternating thick and thin layers. Int. J. Heat Mass Transfer, 26:761-780, 1983. C1116, C1118

[14] D.A. Nield and A. Bejan. Convection in Porous Media. Springer-Verlag, 1992. C1116, C1117, C1120, C1121

[15] I. Pestov. Stability of vapour-liquid counterflow in porous media. J. Fluid Mech., 364:273-295, 1998. C1117

[16] I. Pestov. Modelling non-isothermal flows in porous media: a case study using an example of the Great Artesian Basin, Australia. Proc. Centre for Mathematics and its Applications (R. Melnik, S. Oliveira and D. Stewart, eds.), 38:59-65, 2000. C1119 
[17] K. Pruess. TOUGH2 - A General-Purpose Numerical Simulator for Multiphase Fluid and Heat Flow. LBNL Report LBL-29400, University of California, 1991. C1122

[18] K. Pruess, C. Oldenburg, and G. Moridis. Overview of TOUGH2, Version 2.0. Proc TOUGH Workshop'98, pages 307-315, Lawrence Berkeley National Laboratory, 1998. C1116

[19] P.S. Ramesh and K.E. Torrance. Stability of boiling in porous media. Int. J. Heat Mass Transfer, 33:1895-1908, 1990. C1117

[20] G. Schubert and J.M. Straus. Gravitational stability of water over steam in vapor-dominated geothermal systems. J. Geophys. Res., 85:6505-6512, 1980. C1117

[21] R.A. Wooding. The stability of a viscous liquid in a vertical tube containing porous material. Proc. Roy. Soc. London Ser. A, 252:120-134, 1959. C1117 